\title{
Genetically modified endothelial progenitor cells with hNotch1. ICN overexpression display facilitated angiogenesis
}

\author{
Peng Guo ${ }^{1 \#}$, Hua $\mathrm{Li}^{2 \#}$, Lin Chen ${ }^{3 \#}$, Duo-Ping Wang ${ }^{1}$, Ying Luo ${ }^{2}$, Jian $\mathrm{Xu}^{1}$ \\ ${ }^{1}$ Affiliated Tumor Hospital of Guangxi Medical University, Institute of Cancer Prevention and Treatment of Guangxi Zhuang Autonomous Region, \\ Nanning, China; ${ }^{2}$ College of Stomatology, Guangxi Medical University, Nanning, China; ${ }^{3}$ The First Affiliated Hospital of Guangxi University of \\ Chinese Medicine, Nanning, China \\ Contributions: (I) Conception and design: P Guo; (II) Administrative support: P Guo, J Xu; (III) Provision of study materials or patients: DP Wang; \\ (IV) Collection and assembly of data: H Li, Y Luo; (V) Data analysis and interpretation: L Chen; (VI) Manuscript writing: All authors; (VII) Final \\ approval of manuscript: All authors. \\ "These authors contributed equally to this work. \\ Correspondence to: Peng Guo, PhD; Jian Xu, Professor. Affiliated Tumor Hospital of Guangxi Medical University, Institute of Cancer Prevention and \\ Treatment of Guangxi Zhuang Autonomous Region, Nanning, China. Email: guopeng@stu.gxmu.edu.cn; xjmcf@aliyun.com.
}

Background: This study focused on hNotch1.ICN overexpression and investigated how it affects the biological behavior of endothelial progenitor cells (EPC) in vitro.

Methods: CCK 8 assay was used to evaluate overexpressed hNotch1.ICN to determine how to influence EPCs' survivability. The Annexin V/PI method was used to detect overexpressed hNotch1.ICN and to influence EPC apoptosis. A flow cytometry instrument was used to assess the overexpression of hNotch1.ICN and determine how to influence the EPC cell cycle. Transwell was used to investigate how overexpressed hNotch1.ICN EPCs migrate using their endothelial ability and adhesive ability with activated endothelial cells and angiogenesis ability. After lentivirus gene transfection, qPCR and Western blot were used to detect a notch signaling pathway downstream of the signaling molecules Hes 1 and Hey 1 mRNA and protein expression. The role of the Notch.1 intracellular domain as a candidate EPC regulator with its differential expression and Hes 1 and Hey 1 expression of Notch downstream signaling molecules in separate groups was analyzed.

Results: A detailed analysis revealed an over-expressed hNotch1.ICN gene had no significant effect on canine EPC growth, strengthened EPC antiapoptotic ability, increased numbers of EPCs that underwent cell cycle arrest in the G2 phase, inhibited EPCs differentiation, and enhanced Hes 1 and Hey 1 expression. Moreover, an over-expressed hNotch1 ICN gene promotes EPCs to migrate across ECs, promotes EPCs to adhere to activating endothelial cells, and induces angiogenesis in vitro.

Conclusions: Over-expressed hNotch1.ICN onto EPCs could be used as a potential candidate to treat many ischemic diseases.

Keywords: Endothelial progenitor cell (EPC); Notch signaling pathway; notch 1 intracellular domain; angiogenesis; cell cycle; stem cells differentiation

Submitted Aug 25, 2020. Accepted for publication Oct 11, 2020.

doi: $10.21037 /$ atm-20-6362

View this article at: http://dx.doi.org/10.21037/atm-20-6362

\section{Introduction}

Endothelial progenitor cells (EPCs), also called angioblasts, originate from the blood island of the mesoderm yolk sac and are the precursors of endothelial cells (1). EPCs not only take part in the embryonic stage of angiogenesis but are also involved in angiogenesis after birth. When the body suffers from ischemia, trauma, and stress reactions, or EPCs are induced by cytokines 
or drugs, EPCs in the bone marrow can be stimulated and mobilized into the systemic circulation. These EPCs gradually migrate to the site of ischemia or injury to differentiate into mature endothelial cells and participate in vasculogenesis and angiogenesis in the damaged region (2). Using EPCs as seed cells have broad clinical applications in ischemic diseases, including wound healing, ischemic cerebrovascular disease, and other fields.

The notch signaling pathway plays an important role not only in vascular development but also in the pathological angiogenesis of tumors and the repair processes of tissue injury $(3,4)$. There are Notch signaling pathways and molecular structures on the surface of EPCs (5). The biological behavior of EPCs, including proliferation and migration, is the foundation of the promotion of neovascularization. However, the current mechanism of regulating EPC biological behavior, especially in the notchsignaling pathway, is still not understood. This study set out to over-express hNotch1.ICN in the EPCs, directly activate the notch-signaling pathways and examine the notch pathway's influence on the EPCs biological behavior. We present the following article in accordance with the MDAR reporting checklist (available at http://dx.doi.org/10.21037/ atm-20-6362).

\section{Methods}

The ethics committee approved the study of the Affiliated Tumor Hospital of Guangxi Medical University, Institute of Cancer Prevention and Treatment of Guangxi Zhuang Autonomous Region.

\section{Materials and reagents}

PSB40 (virus control, TU $=7.14 \times 10^{8}$ ), PSB1661 (virus control, TU $\left.=1.02 \times 10^{8}\right)$, primer, and Polybrene were obtained from Sunbio (Shanghai, China). Fetal calf serum and Dulbecco's modified Eagle's medium-high glucose (DMEM-HG) were acquired from Gibco (Grand Island, New York, USA). Trypsin and Trizol were obtained from Invitrogen (Carlsbad, California, USA), while the apoptosis assay kits (FITC/PI) were bought from the Bi Yun Tian Company (Shanghai, China). Antibodies directed against Hes 1 and Hey 1 were acquired from Abcam (Cambridge, MA, USA). Prestained Protein Ladder was obtained from Thermo (Waltham, MA, USA), and TAKARA sold the SYBR Master Mixture (Japan). Transwell was from Millipore (MA, USA). Cell Counting Kit-8 was obtained from the BOSTER Company (Wuhan, China).

\section{Cell culture and transfection}

Canine EPCs were acquired from Shanghai Jing Kang bioengineering company. A human embryonic kidney cell line (293T cells) was acquired from the Shanghai Cell Bank of the Chinese Academy of Sciences. First, the 293T cells were cultured in the DMEM-HG complete medium with $10 \% \mathrm{FBS}, 100 \mathrm{U} / \mathrm{mL}$ penicillin, and $100 \mu \mathrm{g} / \mathrm{mL}$ streptomycin in a humidified atmosphere holding $5 \% \mathrm{CO}_{2}$ at $37^{\circ} \mathrm{C}$. The cells were digested and passaged by $1: 3$ when they reached $>90 \%$ confluence. The $293 \mathrm{~T}$ cells were then transfected using a standard lentivirus method (Invitrogen).

\section{bNotch1.ICN gene overexpressed with lentivirus}

The hNotch1.ICN gene was overexpressed with lentivirus. Gene overexpression technology was used to understand the effects of hNotch1.ICN on the biological behavior of transfected EPCs in vitro. Lentivirus packaging of plasmid pCMV-dR8.9 and pCMV-VSV-G were acquired from Addgene. EPCs were transfected with lentivirus according to the manufacturer's protocol (Sunbio Medical Biotechnology Co., Ltd., Shanghai, China).

\section{Cell counting kit- 8 assay}

EPCs in good condition were respectively digested, counted, and placed into 96 cell cultures, with 2,000 cells per hole. After the cells had adhered to the culture dish (overnight, about 16 hours), according to the MOI $=10$, the purpose and control viruses were respectively added. Simultaneously, $8 \mu \mathrm{g} / \mathrm{mL}$ Polybrene was added to cause an enhanced infection. Then, $10 \mu \mathrm{L}$ CCK- 8 were added to each well 0 hours after infection. Then, the cells were placed in a $37^{\circ} \mathrm{C}$ incubator for 3 hours; then, absorbances at 450 and $620 \mathrm{~nm}$ were measured. The $O D$ values at $450 \mathrm{~nm}$ were measured values, while the OD values at $620 \mathrm{~nm}$ were the values of blanks. Therefore, the final value $=$ measured value-blank value. At specific time intervals following virus infection (24, 48, and 72 hours), the cell supernatant was replaced. Simultaneously, $10 \mu \mathrm{L}$ CCK-8 were added into each well. The cells were put in a $37{ }^{\circ} \mathrm{C}$ incubator for 3 hours, and then their absorbances at 450 and $620 \mathrm{~nm}$ were measured. The final values of the empty cells and the two virus-infected cells were analyzed at four points in time. 


\section{Cell cycle analysis apoptosis assay}

Logarithmic phase EPCs were digested, counted, and placed in three $6-\mathrm{cm}$ dishes, with $6.5 \times 10^{5}$ cells per dish. After being adherent cells (overnight, about 16 hours), according to the MOI $=10$, the purpose and control viruses were respectively added. Simultaneously, $8 \mu \mathrm{g} / \mathrm{mL}$ Polybrene was added to cause an enhanced infection. Twenty-four hours after the virus infection, the cell supernatant was replaced. The culture supernatant was collected 48 hours following virus infection, and the cells were respectively digested with pancreatic enzyme and then collected. The cells were centrifuged for 5 minutes at 1,000 rpm to discard the supernatant. Then, the cells were rinsed with PBS and centrifuged for another 5 minutes at $1,000 \mathrm{rpm}$. The cells were fixed under $4^{\circ} \mathrm{C}$ for 1.5 hours, with $70 \%$ precooling ethanol. The fixed sample was taken out and centrifuged for 5 minutes at 1,000 rpm to abandon any remaining alcohol. The cells were washed with PBS and then resuspended in $400 \mu \mathrm{L}$ precooling RNase PI solution. Afterward, the cell suspension was incubated for 15 minutes at room temperature. The cells were resuspended in $300 \mu \mathrm{L} \mathrm{PBS}$, and the cell cycle was at once analyzed using a flow cytometer. After that, the cells were resuspended in $195 \mu \mathrm{L}$ AnnexinV-FITC and incubated with $10 \mu \mathrm{L}$ PI for 10 minutes at room temperature. Then, the cells were centrifuged to abandon the supernatant and were resuspended in $190 \mu \mathrm{L}$ AnnexinV-FITC and then incubated with $10 \mu \mathrm{L}$ PI for 10 minutes at room temperature. Finally, the cells were respectively resuspended in $100 \mu \mathrm{L}$ PBS and at once analyzed for cell apoptosis using a flow cytometer.

\section{In vitro trans-well migration assay}

In vitro migration of EPCs was performed using transwells. Monolayer ECs were firstly loaded onto trans-well inserts $\left(5 \times 10^{5}\right.$ cells/well). The ECs were cultured and identified in our prior study. When the ECs had been wholly fused, they were then washed three times with PBS. The next step involved the addition of $10 \mathrm{ng} / \mathrm{mL}$ TNFaand $10 \mathrm{ng} / \mathrm{mL}$ IL-to activates the monolayer ECs. Three independent experiments were performed in duplicate. Blank, PSB40, and PSB166 cells were respectively resuspended and loaded onto trans-well inserts $\left(2 \times 10^{5} / \mathrm{mL}\right.$ in serum-free medium). The lower chambers had a $20 \%$ volume fraction serum culture medium. The cells were incubated for 12 hours at $37{ }^{\circ} \mathrm{C}$ and $5 \%$. Then, any cells that migrated to the lower chambers were collected. Five random vision cells with green fluorescence in each transwell were counted.

\section{Adhesion of an activating endothelial cells assay}

ECs were put into 24 cell cultures $\left(1 \times 10^{6}\right.$ per well). When the ECs had fused entirely, they were then washed three times with PBS. Ten $\mathrm{ng} / \mathrm{mL}$ TNF- $\alpha$ and $10 \mathrm{ng} / \mathrm{mL}$ IL$1 \beta$ were used for activating the monolayer ECs. Cell holes were respectively set for Group A, Group B, and Group C. Each group was evaluated in triplicate. Blank, PSB40, and PSB166 cells were uniformly covered onto a monolayer of ECs and incubated for 20 minutes at $37^{\circ} \mathrm{C}$ and $5 \%$. The suspension cells were then washed using PBS, and the numbers of cells were counted in five random fields under green fluorescence.

\section{Angiogenesis}

A tube formation assay was performed to investigate the effect of the hNotch1.ICN gene on the angiogenic activity of EPCs in vitro. Forty-eight-well culture dishes were coated with $150 \mu \mathrm{L}$ of matrigel matrix and incubated for 25 minutes at $37^{\circ} \mathrm{C}$. These cells were divided into blank, PSB40, and PSB166 groups. The cells were starved for 10 hours and were then seeded onto the solidified gels at a density of $10^{6}$ cells/well in $50 \mu \mathrm{L}$ of culture medium. After incubation for 15 hours, the total tube-like structures were photographed using phase-contrast microscopy (100x), and five randomly selected microscopic fields per photograph were quantified using Image J software.

\section{RNA's extraction and quantitative RT-PCR}

The total RNA was extracted from the three groups of cells using Trizol Reagent. First-strand cDNA was synthesized with the PrimeScript Reverse Transcriptase Reagent Kit (Takara Biotechnology, Japan) with oligo (dT) and random primer. The gene expression of the EPCs was quantified using the KAPA Kit (Kapa Biosystems, USA), and $50 \mathrm{ng}$ of cDNA was amplified in a $10 \mu \mathrm{L}$ reaction using the Applied Biosystems 7500 Real-Time PCR (RT-PCR) system with SYBR Green dye (Applied Biosystems). The primers were acquired from Nanjing ChemGen Biotech (Nanjing, China). The amplification primers used were forward primer AGCACAGAAAGTCATCAA and reverse primer TTCTTCAGAGCATCCAAA for the Hes 1 gene. For the 
Page 4 of 10

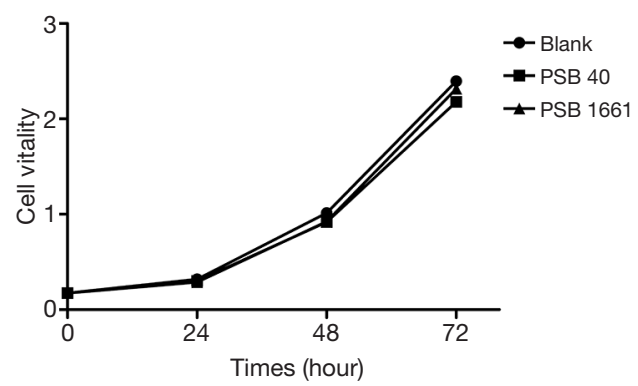

Figure 1 There are no apparent differences in the survival vitality of EPCs in the three groups $(\mathrm{P}>0.05)$. EPC, endothelial progenitor cell.

Hey 1 gene, the amplification primers were forward primer GGATGAGAATGGAAACTTGA and reverse primer ATGATGCCTCTTCGTCTT. QRT-PCR was performed with the following cycling conditions: $95^{\circ} \mathrm{C}$ for 15 seconds, followed by 45 cycles of $95^{\circ} \mathrm{C}$ for 0.05 seconds and $60^{\circ} \mathrm{C}$ for 30 seconds. Quadruplicate cycle threshold (CT) values were analyzed with SDS software (Applied Biosystems, USA) using the comparative CT method. $\beta$-actin was used as an internal control to confirm the success of the reaction. The procedure was repeated three times, and each measurement was set for three repeats.

\section{Western blot analysis}

Total protein lysates from the blank, PSB40, and PSB166 cells were subjected to standard Western blot analysis. The total protein was loaded onto a $4-16 \%$ gradient SDS-PAGE gel and transferred onto a PVDF membrane. Anti-Hey 1 polyclonal antibody (1:500 dilution, Novus) and Anti-Hes1 antibody (1:1,000 dilution, Abcam) were used to detect Hey 1 and Hes 1 protein, respectively. $\beta$-Actin (Santa Cruz) was used as an internal control.

\section{Statistical analysis}

The experimental data were obtained from experiments replicated three times. The Statistical Package for the Social Sciences (SPSS), version 17.0, the software was used for all statistical analyses. Experimental data are shown as mean \pm standard deviations (SD) and have been compared with student's $t$-test or one-way analysis of variance (ANOVA). A $\mathrm{P}$ value $<0.05$ was determined to show statistically significant differences.
Guo et al. hNotch1.ICN overexpression display facilitated angiogenesis

\section{Results \\ No significant differences in growth activity among the three groups}

The survival vitality of EPCs in the three groups had no noticeable difference $(\mathrm{P}>0.05)$, which showed that an overexpressed hNotch1.ICN gene had no significant effect on the EPCs growth (Figure 1).

\section{Overexpressed bNotch1 ICN gene strengthened the EPCs antiapoptotic ability}

Cell apoptosis was detected in infected EPCs after 48 hours using a flow cytometer. The early apoptosis rate of the blank cells was $10.65 \% \pm 0.12 \%$, while the late apoptosis rate was $17.57 \% \pm 0.09 \%$. The early apoptosis rate of PSB 40 cells was $8.29 \% \pm 0.21 \%$, while the late apoptosis rate was $13.85 \% \pm 0.08 \%$. Finally, for PSB1661 cells, the early apoptosis rate was $3.84 \% \pm 0.11 \%$, and the late apoptosis rate was $9.65 \% \pm 0.10 \%$. Both the early and the late apoptosis rates of PSB1661 were lower than the other two groups $(\mathrm{P}<0.05$, Figure 2).

\section{An overexpressed bNotch1.ICN gene increased numbers of EPCs in the G2 phase}

The cell cycle was detected in infected EPCs after 48 hours using flow cytometry. The rates of G1, G2, and S phase in the blank group were $57.63 \% \pm 3.25 \%, 8.60 \% \pm 2.12 \%$, and $33.77 \% \pm 0.12 \%$, respectively. In the PSB 40 group, the rates of $\mathrm{G} 1, \mathrm{G} 2$, and $\mathrm{S}$ phase cells were $65.62 \% \pm 4.11 \%$, $7.47 \% \pm 0.19 \%$, and $27.00 \% \pm 2.58 \%$, respectively. Finally, the G1, G2, and S phase rates in the PSB1661 group were $54.49 \% \pm 3.33 \%, 23.66 \% \pm 2.24 \%$, and $31.86 \% \pm 1.36 \%$, respectively. The numbers of cells in the G2 phase of the PSB1661 group was significantly higher than the other two groups $(\mathrm{P}<0.05$, Figure 3$)$.

\section{An overexpressed bNotch1 ICN gene promoted EPCs to migrate across the ECs}

A migrating cross-vascular endothelial ability reflects the ability of EPCs to form blood vessels. Our experimental results showed that overexpressed hNotch1.ICN gene EPCs of PSB1661 were significantly higher than in the PSB40 and blank groups $(88.1 \pm 11.03$ vs. $31.0 \pm 6.32$ and 

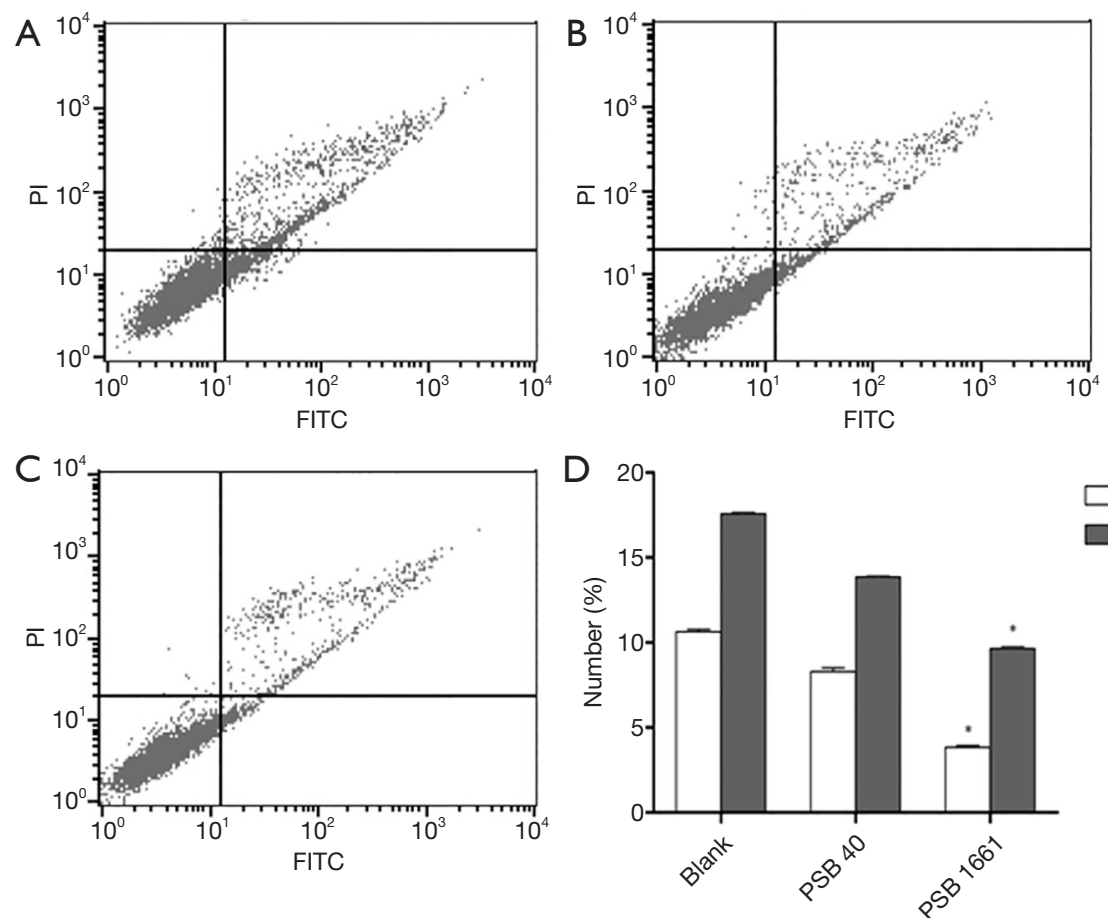

Figure 2 Overexpression of hNotch1.ICN gene can enhance the anti-apoptotic ability of EPCs. (A) Normal EPCs; (B) EPCs transfected with an empty vector; (C) genetically modified EPCs; (D) early and late apoptosis rates were lower for PSB1661 than in the other two groups (*, $\mathrm{P}<0.05)$. EPC, endothelial progenitor cell.
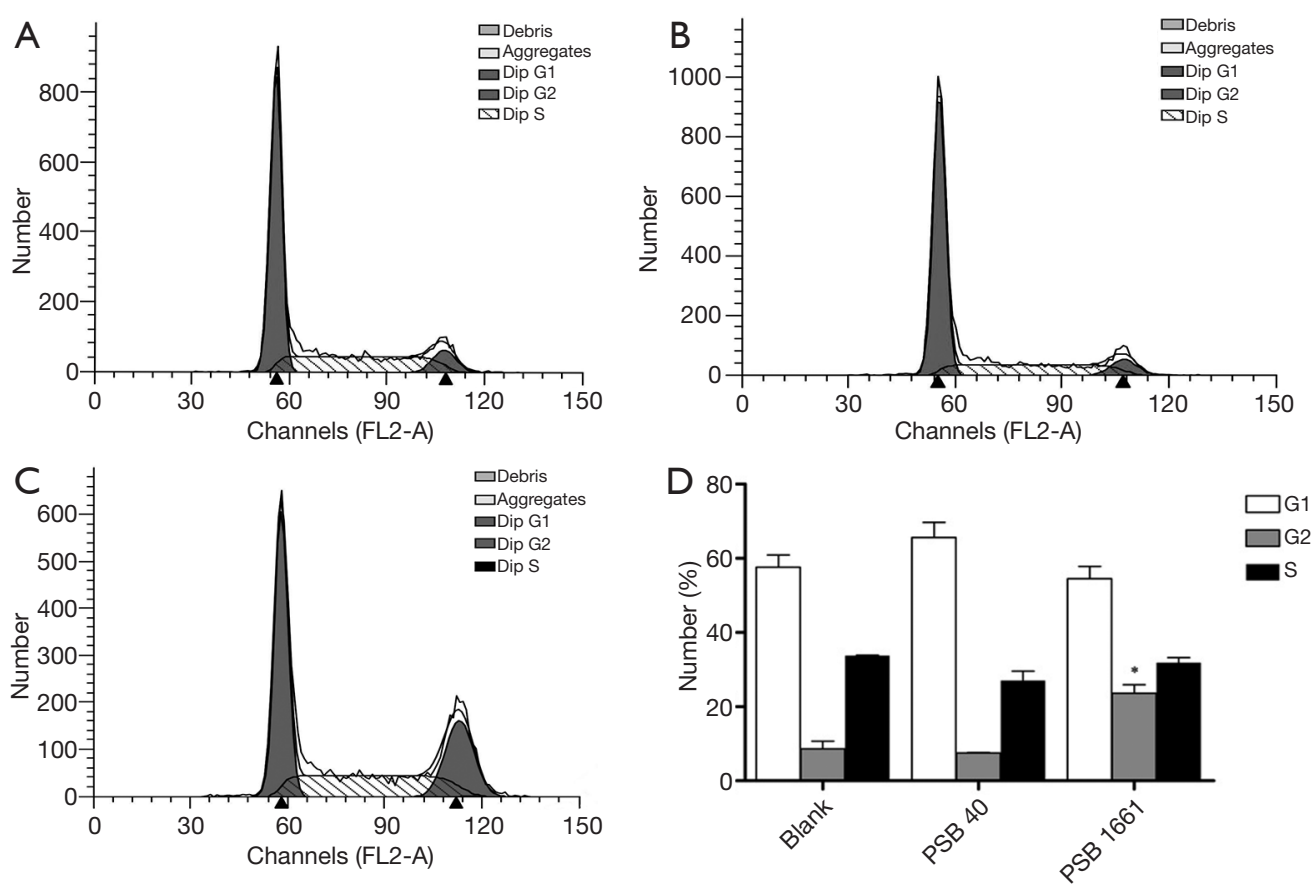

Figure 3 Overexpression of hNotch1.ICN gene increased the number of EPCs in G2 phase. (A) EPCs; (B) EPCs infected with a control virus; (C) infected EPCs with a target virus; (D) numbers of cells in the G2 phase of the PSB1661 group was significantly higher than the other two groups $(*, \mathrm{P}<0.05)$. EPC, endothelial progenitor cell. 

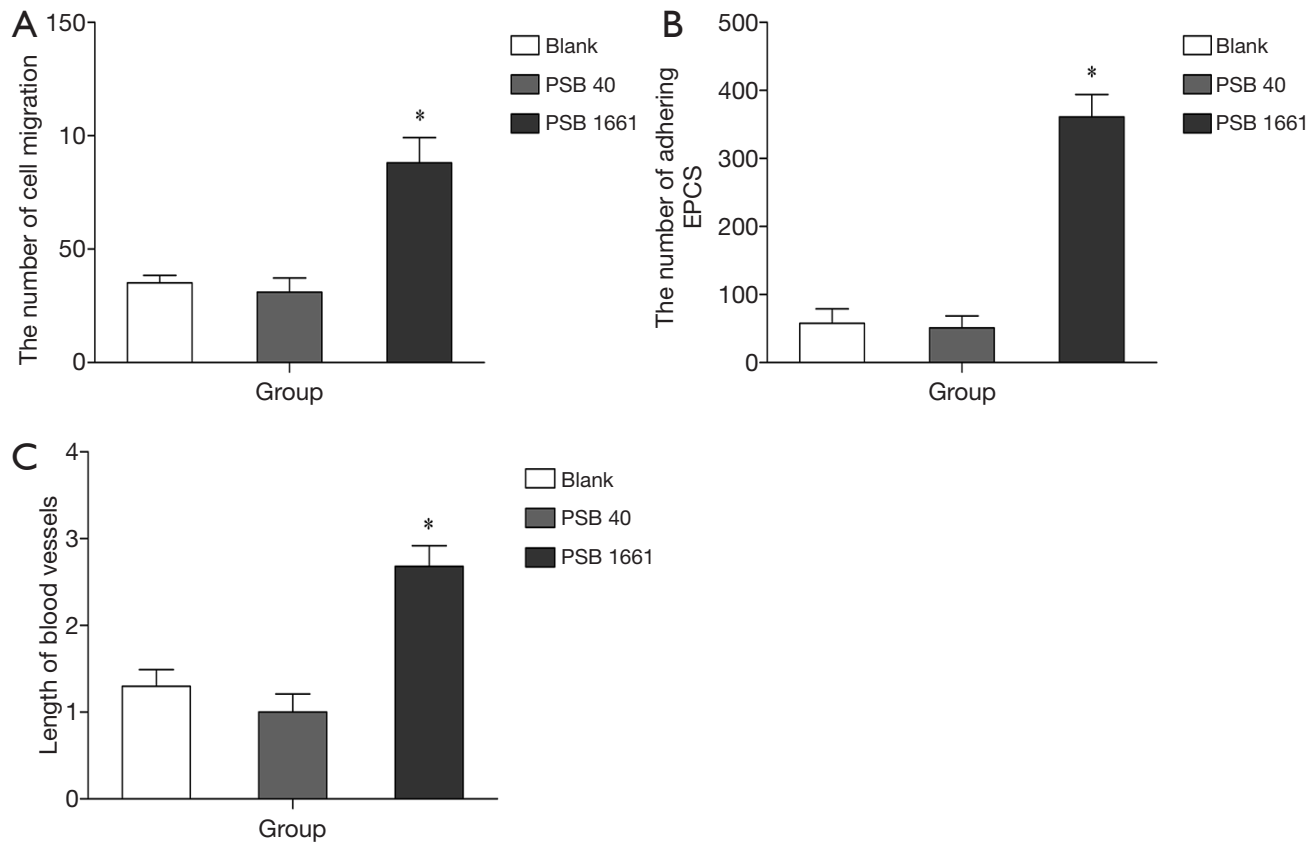

Figure 4 Overexpression of hNotch1.ICN gene can promote EPCs to pass through vascular endothelium, to adhere to activated endothelial cells, and to form lumenoid structure. (A) A comparison of migration across the endothelial cell among three groups ( $x \pm s$, n=3); PSB1661 was significantly higher than the PSB40 or the blank group (*, P<0.05). (B) Comparison of adhesion with activation of endothelial cells among the three groups $(\bar{x} \pm s, \mathrm{n}=3)$, the capacity of PSB1661 cells to adhere to activating endothelial cells was significantly stronger than PSB40, blank cells $\left({ }^{*}, \mathrm{P}<0.05\right)$. (C) A comparison of the forming lumen structure among the three groups $\left(\bar{x}_{ \pm} s, \mathrm{n}=3\right)$. The percentage of PSB1661 cells on matrixforming blood vessels was significantly higher than the other two groups $\left({ }^{*}, \mathrm{P}<0.05\right)$. EPC, endothelial progenitor cell.

35.2 \pm 3.28 , respectively *, $\mathrm{P}<0.05$, Figure $4 A$ ).

\section{An overexpressed bNotch1.ICN gene promoted the EPCs to adhere to activating endothelial cells}

The adherence of EPCs to activating endothelial cells is the crucial step in angiogenesis; it directly affects the time duration of EPCs to stay in the injured or ischemic area and its ability to migrate endothelially. Promoting the EPCs to adhere to activating endothelial cells can significantly enhance the ability of EPCs to form new blood vessels. These experimental results showed that the capacity of PSB1661 cells to adhere to activating endothelial cells was significantly stronger than PSB40 and blank cells $(361 \pm 32.9$ vs. $51.1 \pm 17.6$ and 57.6 \pm 21.5 , respectively *, $\mathrm{P}<0.05$, Figure $4 B)$.

\section{Overexpressed bNotcb1.ICN gene in EPCs induces angiogenesis in vitro}

An in vitro tube cavity structure forming ability is an essential index of the EPCs that participate in angiogenesis. The results showed that overexpression of hNotch1.ICN EPCs onto matrix-forming blood vessels were significantly higher than the other two groups and could effectively improve the ability of EPCs to form a sample tube structure $(2.68 \pm 0.24 v s$. $1 \pm 0.21$ and $1.3 \pm 0.19$, respectively ${ }^{*}, \mathrm{P}<0.05$, Figure $4 C$ ).

\section{An overexpressed bNotch1.ICN gene in EPCs enhances the Hes 1 and Hey 1 expression of Notch downstream signaling molecules}

Overexpression of the hNotch1.ICN gene in PSB1661 cells, as well as Hes 1 and Hey 1 gene expression of the Notch signaling pathways' downstream signaling molecules, were higher than those values for either the PSB40 group or the blank group (*, $\mathrm{P}<0.05)$. The difference was statistically significant (Figure 5). Protein expression was consistent with the results of the expression of genes (Figures 6,7).

\section{Discussion}

EPCs are precursor cells of endothelial cells that are mainly 


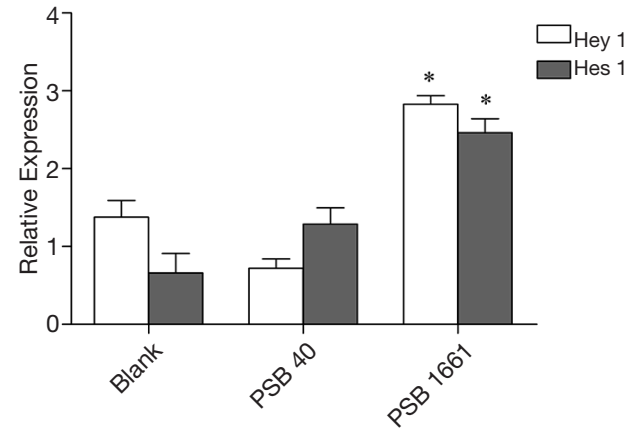

Figure 5 Notch downstream signaling molecules Hes 1 and Hey 1 gene expression. Hes 1 and Hey 1 gene expression rates in the PSB1661 group were higher than those found in the PSB40 group and the blank group $\left(^{*}, \mathrm{P}<0.05\right)$.

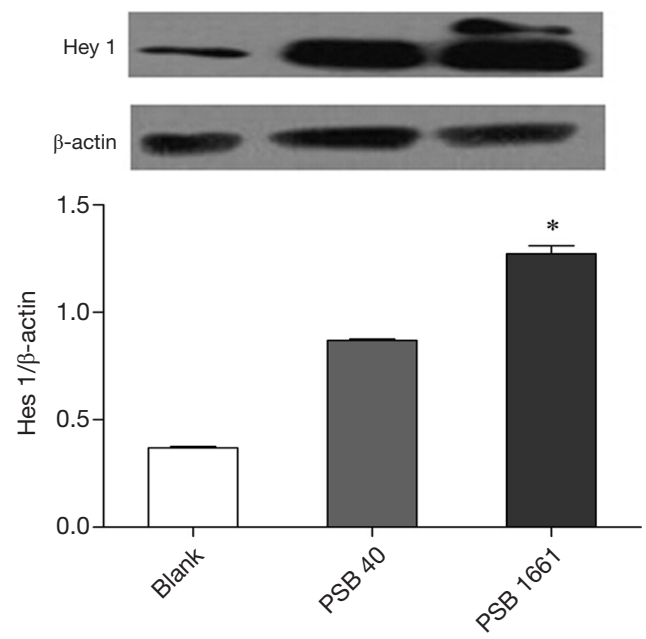

Figure 6 Notch downstream signaling introduces molecule Hes 1 protein expression. The relative gray value of Hes 1 in the PSB1661 group was higher than the other two groups $\left({ }^{*}, \mathrm{P}<0.05\right)$.

derived from bone marrow (6), but a few may also be found in umbilical cord blood, peripheral blood, and myocardial tissue (7-9). EPCs play an essential role in angiogenesis and endothelial repair (10). In many ischemic animal research models, EPCs from umbilical cord blood, peripheral blood, or bone marrow can cure or even alleviate ischemia $(11,12)$. The angiogenesis process includes several steps that involve EPCs. Silent bone marrow EPCs are activated, mobilized into the peripheral blood circulation, and finally remain in the area of tissue ischemia to differentiate into endothelial cells in situ to form new blood vessels (13). Because they are directly involved in angiogenesis, EPCs can also have a

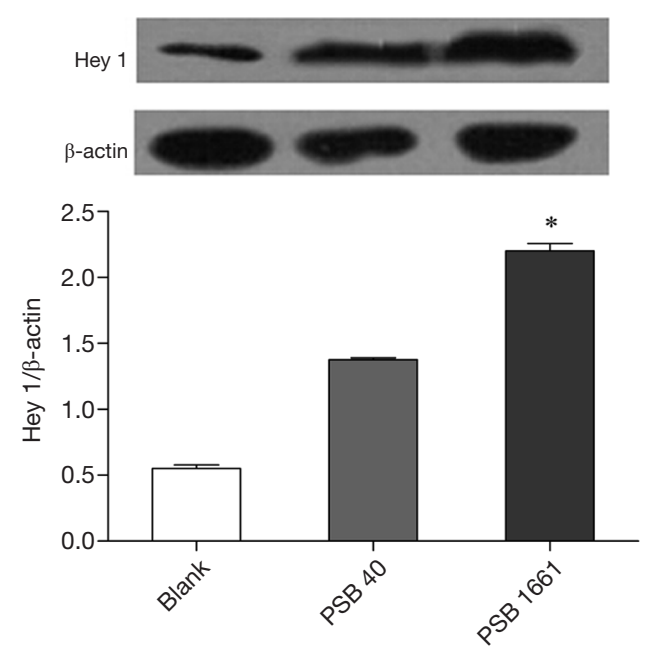

Figure 7 Notch downstream signaling introduces molecule Hey 1 protein expression. The relative gray value of Hey 1 in the PSB1661 group was higher than the other two groups $\left(^{*}, \mathrm{P}<0.05\right)$.

chemotaxis effect on vascular defect points and promote the formation of new blood vessels. Many studies have found that EPCs can promote angiogenesis. For example, in tissue engineering, EPCs are used as vascularized seeds to promote bone vascularization and improve osteogenesis (5). For repairing bone defects, EPCs combined with the tissueengineered bone are better than simplex tissue-engineered bone (14). The functions of EPCs are closely related to their biological characteristics. In general, EPCs are resting and can also support an immature state. However, when a stimulus induces EPCs or some signal molecule that can adjust for angiogenesis, EPCs will migrate, proliferate, and differentiate into mature endothelial cells. These mature endothelial cells promote new blood vessel formation to repair the damaged vasculature (15).

Many signaling pathways regulate EPCs that take part in angiogenesis. Many studies have shown that notch signaling plays a crucial role in regulating cell differentiation and angiogenesis. Recent studies have shown the characteristics of vascular endothelial cell differentiation towards arteriovenous are mainly regulated by notch signaling molecules (16), and the notch signaling pathway has a close relationship with smooth muscle cell differentiation and tumor angiogenesis. The notch signaling pathway is made up of notch ligands, receptors, and effector molecules into cells (17). When notch receptors combine with ligands, the notch signaling pathway is activated, the Notch intracellular domain (Notch.ICN) is released, and then 
Notch.ICN transfers into the cell nucleus and activates the downstream effector molecules, which inhibit the differentiation and transcription of related genes. Notch. ICN codes Notch 1 proteins across the membrane area and in front of the PEST structure domain. The fragments of ICN have a complete ARM and ANK structure domains. These domains activated Notch 1 directly through the notch signaling pathway in the absence of ligands. The notch signaling pathway plays an important signal transduction role in the interaction between cells and stem cell self-renewal, proliferation, adhesion, apoptosis, and migration $(18,19)$.

To study how Notch signaling molecules influence the biological characteristics of EPCs, we adopted a gene transfection method. Genetic modification enhances the function of EPCs. EPCs can be used as target cells of gene therapy, and they also produce therapeutic proteins at the site of injury. This result showed that the overexpression of the hNotch1.ICN gene had no significant impact on the activity or proliferation ability of EPCs. There was no apparent difference among the blank group, the PSB40 group, and PSB1661 cells.

The notch signaling pathway may be crucial in supporting the undifferentiated state of progenitor cells. Duncan observed that in the bone marrow and peripheral blood, notch activity was down-regulated when hematopoietic progenitor cells differentiated (20). To verify this conclusion, the researchers used interleukin 3 and steel factor cultured KLS GFP ${ }^{+}$cells. Three days later, KLS GFP ${ }^{+}$cells were differentiated into Lin+ cells. The result of $13 \%$ Lin + cells that expressed GFP, which they addressed the finding of $12 \%$ obtained before Lin's cell differentiation, and the GFP fluorescence density in Lin- cells was lower. These results showed that an activated notch signal decreased the differentiation, and the fluorescent signal level also decreased. YU also found the Hes 1 gene, the primary target molecule of Notch signaling pathways downstream, was highly expressed in the CD34+, CD38-, and Lin- cells but was lowly expressed in more mature CD34+ and CD38+ cells. In this study, overexpression of the hNotch1.ICN gene caused the Notch signaling pathway to be continuously activated (21). We found Hes 1 and Hey 1, which are Notch signaling downstream effector molecules, increased their expression. Hes 1 and Hey 1 inhibited specific target gene transcription.

Additionally, overexpression of the hNotch1.ICN gene increased numbers of EPCs that underwent cell cycle arrest in the G2 phase, delayed the cell cycle's progress, reduced the differentiation velocity of EPCs, and inhibited the differentiation of stem cells. In this study, we used the Annexin V/PI method to detect a blank group, PSB40 group, and PSB1661 group cells. We found the early and the late apoptosis rates of PSB1661 were $3.84 \% \pm 0.11 \%$ and $9.65 \% \pm 0.10 \%$, respectively, which was lower than the other two groups. The results showed that overexpression of the hNotch1.ICN gene enhanced bone marrow EPCs' ability to resist apoptosis, inhibited hematopoietic progenitor cell differentiation, and contributed a long-term recovery effect.

EPCs can gather, adhere, stay in the vascular injury area, proliferate, differentiate, and secrete cytokines, and finally repair damaged endothelial cells or blood vessels. Adhesion and migration are essential links in EPC homing. In this study, by simulation of the existence, movement, and the interaction of cells with the endothelial microenvironment, we described EPCs involvement in essential steps of angiogenesis. The experimental results showed that overexpression of the hNotch1.ICN gene can significantly enhance the ability of EPCs to adhere to activated endothelium, migrate across the endothelium, and improve EPC tube structure formation in vitro.

This work indicated that overexpression of the hNotch1.ICN gene can significantly enhance the ability of canine bone marrow EPCs to resist apoptosis, inhibit hematopoietic progenitor cell differentiation, and help EPCs to exert a long-term recovery effect. Overexpression of the hNotch1.ICN gene can enhance the angiogenesis ability of canine EPCs and supply a new strategy for EPCs as seed cells to treat many types of ischemic diseases.

\section{Acknowledgments}

Funding: This work was funded by grants from the Natural Science Foundation of Guangxi Province of China (General Program: 2018GXNSFAA281215), Guangxi Province Natural Science Foundation for Youths (2017GXNSFBA198171), The Basic Ability Enhancement Program for Young and Middle-aged Teachers of Guangxi (2018KY0101).

\section{Footnote}

Reporting Checklist: The authors have completed the MDAR reporting checklist. Available at http://dx.doi.org/10.21037/ atm-20-6362

Data Sharing Statement: Available at http://dx.doi. 
org/10.21037/atm-20-6362

Conflicts of Interest: All authors have completed the ICMJE uniform disclosure form (available at http://dx.doi. org/10.21037/atm-20-6362). The authors have no conflicts of interest to declare.

Ethical Statement: The authors are accountable for all aspects of the work in ensuring that questions related to the accuracy or integrity of any part of the work are appropriately investigated and resolved. The ethics committee approved the study of the Affiliated Tumor Hospital of Guangxi Medical University, Institute of Cancer Prevention and Treatment of Guangxi Zhuang Autonomous Region.

Open Access Statement: This is an Open Access article distributed in accordance with the Creative Commons Attribution-NonCommercial-NoDerivs 4.0 International License (CC BY-NC-ND 4.0), which permits the noncommercial replication and distribution of the article with the strict proviso that no changes or edits are made and the original work is properly cited (including links to both the formal publication through the relevant DOI and the license). See: https://creativecommons.org/licenses/by-nc-nd/4.0/.

\section{References}

1. Huang HT, Liu ZC, Wu KQ, et al. MiR-92a regulates endothelial progenitor cells (EPCs) by targeting GDF11 via activate SMAD2/3/FAK/Akt/eNOS pathway. Ann Transl Med 2019;7:563.

2. Aguirre A, Planell JA, Engel E. Dynamics of bone marrow-derived endothelial progenitor cell/mesenchymal stem cell interaction in co-culture and its implications in angiogenesis. Biochem Biophys Res Commun 2010;400:284-91.

3. Pedrosa AR, Trindade A, Carvalho C, et al. Endothelial Jagged1 promotes solid tumor growth through both pro-angiogenic and angiocrine functions. Oncotarget 2015;6:24404-23.

4. Djokovic D, Trindade A, Gigante J, et al. Incomplete Dll4/Notch signaling inhibition promotes functional angiogenesis supporting the growth of skin papillomas. BMC Cancer 2015;15:608.

5. Keramaris NC, Kaptanis S, Moss HL, et al. Endothelial progenitor cells (EPCs) and mesenchymal stem cells (MSCs) in bone healing. Curr Stem Cell Res Ther
2012;7:293-301.

6. Goligorsky MS. Endothelial progenitor cells: from senescence to rejuvenation. Semin Nephrol 2014;34:365-73.

7. Sheng ZL, Yao YY, Li YF, et al. Transplantation of bradykinin-preconditioned human endothelial progenitor cells improves cardiac function via enhanced Akt/eNOS phosphorylation and angiogenesis. Am J Transl Res 2015;7:1214-26.

8. Abd El Aziz MT, Abd El Nabi EA, Abd El Hamid M, et al. Endothelial progenitor cells regenerate infracted myocardium with neovascularisation development. J Adv Res 2015;6:133-44.

9. Eggermann J, Kliche S, Jarmy G, et al. Endothelial progenitor cell culture and differentiation in vitro: a methodological comparison using human umbilical cord blood. Cardiovasc Res 2003;58:478-86.

10. Laurenzana A, Fibbi G, Margheri F, et al. Endothelial Progenitor Cells in Sprouting Angiogenesis: Proteases Pave the Way. Curr Mol Med 2015;15:606-20.

11. Murohara T, Ikeda H, Duan J, et al. Transplanted cord blood-derived endothelial precursor cells augment postnatal neovascularization. J Clin Invest 2000;105:1527-36.

12. Asahara T, Masuda H, Takahashi T, et al. Bone marrow origin of endothelial progenitor cells responsible for postnatal vasculogenesis in physiological and pathological neovascularization. Circ Res 1999;85:221-8.

13. Sainz J, Sata M. CXCR4, a key modulator of vascular progenitor cells. Arterioscler Thromb Vasc Biol 2007;27:263-5.

14. Atesok K, Matsumoto T, Karlsson J, et al. An emerging cell-based strategy in orthopaedics: endothelial progenitor cells. Knee Surg Sports Traumatol Arthrosc 2012;20:1366-77.

15. Sun R, Zhang J, Xiong M, et al. Altered Expression of Genes in Signaling Pathways Regulating Proliferation of Hematopoietic Stem and Progenitor Cells in Mice with Subchronic Benzene Exposure. Int J Environ Res Public Health 2015;12:9298-313.

16. Gehling UM, Ergun S, Schumacher U, et al. In vitro differentiation of endothelial cells from AC133-positive progenitor cells. Blood 2000;95:3106-12.

17. Li JL, Harris AL. Notch signaling from tumor cells: a new mechanism of angiogenesis. Cancer Cell 2005;8:1-3.

18. Su BH, Qu J, Song M, et al. NOTCH1 signaling contributes to cell growth, anti-apoptosis and metastasis in salivary adenoid cystic carcinoma. Oncotarget 2014;5:6885-95. 
19. Farnie G, Clarke RB. Mammary stem cells and breast cancer--role of Notch signalling. Stem Cell Rev 2007;3:169-75.

20. Duncan AW, Rattis FM, DiMascio LN, et al. Integration of Notch and Wnt signaling in hematopoietic stem cell maintenance. Nat Immunol 2005;6:314-22.

Cite this article as: Guo P, Li H, Chen L, Wang DP, Luo Y, $\mathrm{Xu}$ J. Genetically modified endothelial progenitor cells with hNotch1.ICN overexpression display facilitated angiogenesis. Ann Transl Med 2020;8(20):1316. doi: 10.21037/atm-20-6362
21. Yu X, Alder JK, Chun JH, et al. HES1 inhibits cycling of hematopoietic progenitor cells via DNA binding. Stem Cells 2006;24:876-88.

(English Language Editor: J. Chapnick) 